\section{PSICOLOGIA IBEROAMERICANA}

\section{Psicología lberoamericana}

ISSN: 1405-0943

psicología.iberoamericana@uia.mx

Universidad Iberoamericana, Ciudad de

México

México

González Jaimes, Elvira Ivone; Pérez Saucedo, Eduardo Factores de Riesgo Laboral y la Salud Mental en Trabajadores de la Salud Psicología Iberoamericana, vol. 19, núm. 2, julio-diciembre, 2011, pp. 67-77

Universidad Iberoamericana, Ciudad de México

Distrito Federal, México

Disponible en: http://www.redalyc.org/articulo.oa?id=133921440008

Cómo citar el artículo

- Número completo

- Más información del artículo

- Página de la revista en redalyc.org

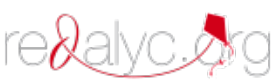

Sistema de Información Científica

Red de Revistas Científicas de América Latina, el Caribe, España y Portugal Proyecto académico sin fines de lucro, desarrollado bajo la iniciativa de acceso abierto 


\title{
Factores de Riesgo Laboral y la Salud Mental en Trabajadores de la Salud
}

\author{
Labor Risk Conditions and Mental Health in Workers from Health
}

\author{
Elvira Ivone González Jaimes* \\ Centro Universitario UaEM \\ Universidad Autónoma del Estado de México \\ Eduardo Pérez Saucedo* * \\ Departamento de Salud Mental, Hospital General del IMSS, CD. de México
}

\section{RESUMEN}

Se realizó un estudio sobre factores de riesgo laborales y su repercusión en la salud mental en trabajadores de este sector. Se investigaron correlaciones significativas entre los resultados en cuatro grupos de factores:1) clima organizacional, 2) motivación, 3) motivación y satisfacción laboral, 3) desgaste profesional y 4) trastorno por consumo de alcohol. Se utilizó un diseño no experimental, tipo post-facto, transversal, descriptivo, analítico y de campo en formato de encuesta, con una muestra aleatoria de 5662 trabajadores pertenecientes a 20 hospitales del sector salud de la Ciudad de México y el Estado de México. Material, Test Clima Organizacional, Test Motivación y Satisfacción Laboral, Maslach Burnout Inventory y Alcohol Use Disorders Identification Test. Se observaron trabajadores con conflictos en clima laboral, 35\%, baja motivación e insatisfacción laboral, 32.9\%, síndrome de Burnout, 27.3\%, y trastorno por consumo de alcohol, 7.2\%. Se concluyó que los trabajadores que presentaron baja motivación y conflictos laborales tenían más probabilidades de padecer desgaste profesional y consumo de alcohol en riesgo.

Descriptores: Clima organizacional, motivación y satisfacción laboral, desgaste profesional y trastorno por consumo de alcohol.

\section{ABSTRACT}

Labor risk conditions and its repercussion in mental health factors of health sector workers. Investigation of significant correlation that exists between result of four groups of tests, that measure: Labor condition factors and mental health factors. Non experimental Design, transversal post-facto type, descriptive, analytical and survey format, Applied to 5,662 workers of 20 hospitals from health sector in Mexico City and Mexico State. Material, Organizational environment Test, Motivation and Labor Satisfaction Test, Maslach Burnout Inventory and Alcohol Uses Disorders Identification Test.

Results. Workers with: conflicts in labor climate $35 \%$, low motivation and labor dissatisfaction $32.9 \%$, Burnout syndrome $27.3 \%$, and alcohol use disorder $7.2 \%$.

Conclusions, Workers that have low motivation and labour conflicts present frequently professional depletion and risk alcohol use.

Key words: Organizational climate, motivation and labor satisfaction, professional depletion and alcohol use disorders

\footnotetext{
* Para correspondencia: Módulo de Investigación, Centro Universitario UAEM, Valle de México, Universidad Autónoma del Estado de México. Bulevar Universitario s/n, Predio San Javier, Municipio de Atizapán de Zaragoza, Estado de México, 54500. Teléfonos: $53791745,0445513406950$. Fax: 58270703. Correo electrónico: ivonegj@hotmail.com

** Mtro. en Psicología. Para correspondencia: Módulo de Investigación del Departamento de Salud Mental, Hospital General, Centro Médico La Raza, IMSS, México, D. F. Correo electrónico: ejaimes@itesm.mx
} 


\section{INTRODUCCIÓN}

Desde 1984, el Comité Mixto de la Organización Internacional del Trabajo (OIT)-Organización Mundial de la Salud (OMS) ha puesto de relieve que los factores de riesgo psicosocial en el trabajo abarcan las interacciones entre el medio ambiente laboral y la salud mental. Ante estas iniciativas mundiales, se han realizado estudios sin establecer etiologías generales de los mismos, dado su carácter multifactorial.

Continuando con esta iniciativa, en 2007, la OMS, trazó un "Plan de acción mundial sobre la salud de los trabajadores 2008-2017, con el propósito de establecer un marco normativo que permita la adopción de medidas concertadas destinadas a proteger, promover y mejorar la salud de todos los trabajadores".

Por ello, el presente estudio aborda la relación entre las condiciones laborales y su repercusión en factores de salud mental en trabajadores del sector salud, para poder determinar cuáles de éstas se pueden jerarquizar como factores de riesgo en el trabajo.

¿Por qué a trabajadores del sector salud? Porque éstos deben poseer una adecuada salud física y mental para la labor que tienen encomendada, que es "la salud".

Existen estudios que relacionan las condiciones físicas laborales son proporcionales con el presupuesto que manejan el ámbito laboral (Álvarez, 1992) y otros, que refieren que las condiciones físicas afectan a factores emocionales (González \& López, 2009).

El presente estudio se realizó en el Instituto Mexicano del Seguro Social (IMSS) por dos razones: porque éste opera con déficit presupuestario desde la década de los ochenta, lo que ha alterado su capacidad de prestación de servicio médico, y porque se le considera la institución de salud más grande de América Latina.

Según el Informe de la Evaluación Especifica de Desempeño 2010-2011, el IMSS presta servicio médico a 43871139 derechohabientes $^{1}$ a través de 300000 trabajadores de "base,"2 cifra que fluctúa según la contratación.Dicha institución presenta un déficit presupuestal que ha provocado una serie de reajustes estructurales apoyados por las nuevas políticas de globa-

\footnotetext{
${ }^{1}$ Son las personas aseguradas y sus beneficiarios protegidos por el régimen ordinario y régimen voluntario del IMSS.

${ }^{2}$ Trabajadores con contratación definitiva y sindicalizados.
}

lización, como se muestra en la "Campaña Mundial en Materia de Seguridad Social y Cobertura para Todos", promovida por la OIT (2002); dicha campaña se utilizó como medida para resolver problemas económicos y la desigualdad de cobertura seguridad social. Se focalizó en los empresarios y en los trabajadores de la economía formal, quienes están obligados a sostener el sistema de seguridad social a través de contribuciones económicas e impuestos; mientras los trabajadores de la economía informal no contribuyen al pago de la seguridad social (OIT, 1993).

En América Latina, se propuso la privatización de la seguridad social a través de la responsabilidad individual y la propiedad directa de los ahorros provisionales para solventar la cobertura.

Este rediseño pretende: 1) aumentar los incentivos de trabajadores para que se adhieran, permanezcan y contribuyan en los sistemas de salud; 2) reducir el empleo informal y el sub-registro laboral. Sin embargo, estas reformas de ingreso a los sistemas de salud privado, en vez de lograr la universalidad o aumentar la cobertura, lo han reducido (Savio, 2008).

Los puntos a estudiar son: 1) clima organizacional, 2) motivación y satisfacción laboral, 3) desgaste profesional o Síndrome de Burnout (SB), y 4) Trastorno por Consumo de Alcohol (TCA).

Se hizo un estudio de los factores que pudieran afectar las condiciones laborales:

1) Disminución del mantenimiento en condiciones físicas laborales y de materiales básicos para la realización del trabajo, debido a: 1.1) el aumento, en la última década, de " $5 \%$ por año en gastos médicos", como lo reporta la Organización para la Cooperación Económica y el Desarrollo (OECD, 2007); 1.2) la reducción del presupuesto indicado en la reforma a la Ley del Seguro Social (última reforma publicada: DOF 09-07-2009,1995), donde se redujo la cuota patronal a la institución de seguridad social en $33 \%$, lo que significó la pérdida de 35 millones de pesos diarios para la institución, es decir, 12775 millones anuales; 1.3) recorte del presupuesto en 1780 millones de pesos, expresado en el Presupuesto de Egresos de la Federación en la Ley del Seguro Social (última reforma publicada: DOF 09-07-2005). Esto ha dado como resultado "un déficit superior a un tercio del Producto Interno Bruto de 2006, más de 33\%, una cifra más bien cercana a 
$36 \%$ del Producto Interno Bruto"; lo que en pesos significa "un descuadre de 17000 millones". Estos índices fueron mencionados en la última comparecencia del director del IMSS, Juan Molinar Horcasitas, el 24 abril 2007 (Transcripción de la Comparecencia del Director General del IMSS, ante la Comisión de Seguridad Social de la Cámara de Diputados, 2007).

En 2010 se recibió $13.9 \%$ menos de recursos para inversión, al asignársele al IMSS un presupuesto de 6488 millones de pesos, según el último Informe Financiero y Actuarial del IMSS, y el Proyecto de Presupuesto de Egresos 2010 (Agencia Reforma, 2009).

2) Sobrecarga de trabajo debido a: 2.1) incremento de derechohabientes con enfermedades crónicas degenerativas y el sector de la tercera edad (transición epidemiológica); dicho aumento ha ocurrido hasta en $300 \%$ en los últimos 15 años (Lara, Benítez, Fernández \& Zárate, 1996); 2.2) disminución en la creación de 17500 nuevas plazas debido a la eliminación de la bilateralidad en la contratación colectiva; reforma realizada a la Ley del Seguro Social, artículo 277 D, publicado en el Diario Oficial de la Federación del 11 de agosto de 2004 (Ley del Seguro Social, 2004); 2.3) aumento de la frecuencia de ocupación de camas debido a la poca capacidad de atención hospitalaria, que es de "0.86 camas por cada mil derechohabientes", según reporte del director general esta institución de salud; en el cual se reconoce también que: "es un número inaceptable para un país perteneciente a Organización para la Cooperación Económica y el Desarrollo, y muy bajo para un país, incluso de desarrollo inferior al nuestro." (Transcripción de la Comparecencia del Director General del IMSS, Maestro Juan Molinar Horcasitas, ante la Comisión de Seguridad Social de la Cámara de Diputados, 2007).

Con esta visión general del deterioro económico del IMSS, valoraremos:

a) El clima organizacional, ya que, según Molinar (2007), en él se refleja la historia de luchas internas y externas de poder y la jerarquía entre los diferentes organismos que gobiernan. "Lo que hace crecer a las organizaciones son buenos o malos manejos de los recursos humanos que son orientados hacia líneas de trabajo acordes a los intereses de las autoridades en el poder" (Álvarez, 1992).
Otros autores (Carr, Schmidt \& Deshon, 2003; Parker et al., 2003) comentan que el clima organizacional influye en la salud física y mental de los trabajadores. Observaron que, cuando éste es positivo provee logros, afiliación, poder, productividad, baja rotación y satisfacción, mientras que, cuando es negativo, se manifiesta por medio de inadaptación, alta rotación, ausentismo y baja productividad.

b) La motivación laboral, definida por González (2000) como "la fuerza que requiere un trabajador para emprender con éxito su actividad productiva". Este autor, en un estudio para encontrar la etiología de la motivación laboral, la dividió en tres ejes básicos: 1) programa de pagos de incentivos, beneficio importante para el trabajador cuando desarrolle correctamente aspectos importantes del puesto; 2) enriquecimiento del puesto, o incremento de la motivación intrínseca de los trabajadores con incentivos emocionales ante conductas asertivas laborales; 3 ) administración por objetivos, o sea, estimular la creación de nuevos valores para lograr los objetivos estratégicos de la organización.

La satisfacción laboral es considerada por diferentes teóricos e investigadores en ciencias sociales como "factor determinante de la calidad de la atención médica." Esta aportación se aplica frecuentemente en los modelos de evaluación de los servicios de salud (Hernández, Dickson \& Fernández, 2008).

c) El desgaste profesional o Sindrome de Burnout $(S B)$, que Freudernberger (1974) define como un "estado de fatiga o de frustración que se produce por la dedicación a una causa, forma de vida o de relación, desgastando a largo plazo la estructura física y emocional". Esta circunstancia se ha descrito metafóricamente como "el síndrome del quemado", por el alto desgaste que sufren los profesionales de la salud.

Estudios previos han demostrado que el SB es un desgaste profesional acelerado en trabajadores de salud insatisfechos, que provoca poca sensibilidad y comprensión, "manifestando a veces conductas agresivas hacia los pacientes, con un trato distanciado y cínico como tendencia a culpar al paciente de sus propios problemas" (Álvarez, 2009). "Deteriorando con el tiempo la calidad de servicio que se brinda", según comentario de la Sociedad Médica de Urgencia, Barcelona España (Belloch-G, Calabuig, GómezSalinas y Renovell, 2000). 
En resguardo de la integridad física y mental de los trabajadores, el SB fue adoptado por los sindicatos y abogados como elemento de ayuda para los problemas físicos generados por un grado de agotamiento excesivo. En la actualidad es una de las causas más importante de incapacidad laboral (Caraveo, Ramírez, Villatoro, Richerand y Escobar, 1988).

Existen varios estudios en población mexicana sobre la prevalencia y los factores asociados al Burnout en áreas de salud. Se han detectado alteraciones en las subescalas: cansancio emocional, despersonalización y falta de realización personal (Franco, 1999; López, Rodríguez, López, Peralta y Munguía, 2007).

En un estudio de Pardo, López, Moriña, Pérez, Freire y Fernández (2002), 45.26\% de los trabajadores de la muestra presentaba alteraciones físico-psíquicas relacionadas con su trabajo; $65.11 \%$, ansiedad-estrés; $13.95 \%$ cefaleas; $13.95 \%$, afectaciones osteoarticulares, y depresión, 6.97\%. Además, consideraban que sus problemas laborales afectaban su vida familiar en "nada", 29.47\%; "un poco", 30.52\%; "moderadamente", $32.63 \%$; y "bastante", 4.2\%. Ortega y López (2004) realizaron un estudio teórico exhaustivo, en donde reflexionaron sobre la etiología y el manejo del SB en profesionales sanitarios. Informaron sobre las frecuentes medidas y estrategias utilizadas para la prevención y la curación de este padecimiento, destacando las funciones económico-administrativas de los centros de trabajo, ya que de ellos depende el buen o mal funcionamiento de las instituciones y su personal.

d) Trastorno por Consumo de Alcohol (TCA). Existen investigaciones que relacionan las malas condiciones de trabajo con la dependencia y el abuso del alcohol. Un estudio realizado con trabajadores mexicanos registra que $26.5 \%$ beben alcohol en riesgo, y 5.3\% beben en riesgo peligroso (Carreño, MedinaMora, Martínez, Juárez, y Vázquez, 2006).

El TCA repercute en ambientes laborales, tanto por el bajo desempeño laboral, faltas injustificadas y accidentes de trabajo, como en la salud integral de los trabajadores (Medina-Mora y Echeverría, 2005).

La Encuesta Nacional de Adicciones (ENA, 2002) demuestra que el consumo de bebidas alcohólicas ocurre con más frecuencia en las etapas productivas de la vida, asegurando que "la mayor parte de los bebedores fuertes y de los dependientes están integra- dos a la fuerza de trabajo". Asimismo, refiere que el porcentaje de los trabajadores que tienen problemas laborales es de $3.7 \%$, y con pérdida del empleo o en posibilidad de perderlo de $1.4 \%$. Como se esperaba, la población que calificó para el trastorno abuso/dependencia tiene más problemas laborales que aquellos que no presentan dicho trastorno.

Esta misma encuesta, unos años después (ENA, 2008), indica que el abuso/dependencia al alcohol es muy elevado. Hace hincapié en que 4168063 cumple con los criterios para este trastorno; de éstos, 3497946 son hombres y 670117 , mujeres.

El TCA es detonador de conflictos laborales, pérdida de años de vida saludable, morbilidad, mortandad, alto costo social, entre otros factores que perjudican la calidad de vida del ser humano en edad productiva (Hernández, Dickson y Fernández, 2008).

Estudios realizados en empresas que pertenecen a la OCDE (2007) reportan que, de cada 100 trabajadores tres sufren TCA. Según estas investigaciones, "los trabajadores alcohólicos consuetudinarios y recurrentes altos presentan ausencias y retardos laborales en una proporción dos veces y media más que otros trabajadores, y los trabajadores con abuso de bebidas alcohólicas reciben incapacidades tres veces más que otros trabajadores".

Por la alta prevalencia del consumo de alcohol dentro de esta institución, se ha modificado el Contrato Colectivo (Contrato Colectivo de Trabajo del Instituto Mexicano del Seguro Social, 2007-2009) en su cláusula 40 (Faltas justificadas con posterioridad), para que los trabajadores que padezcan TCA ingresen a programas de tratamiento contra su adicción.

\section{MÉTODO}

Diseño no experimental, transversal, descriptivo, de campo, en formato de encuesta (Rojas, 1998).

\section{Participantes}

La muestra fue de 18434 trabajadores encuestados de "base" (con contrataciones definitivas y sindicalizados) de seis categorías, de ambos géneros, de 18 a 60 años de edad, pertenecientes a los 20 hospitales del Distrito Federal y el Estado de México (escogidos al azar).

El cálculo del tamaño de la muestra fue determinado con base en el tema para diseño de la muestras 
en estudios complejos (Rojas, 1998), tomando como $\mathrm{pq}=.31$ la prevalencia promedio de conflictos laborales reportado por la OIT.

El muestreo, teniendo ya el tamaño $(n=5,662)$, seleccionó al azar las encuestas contestadas de los trabajadores de "base" requeridos.

Se utilizó un análisis estadístico de tipo descriptivo para conocer la distribución de las variables de la muestra y dos de tipo inferencial (Chi- cuadrada de Pearson $\left.\mathrm{p} \leq 0.05 ; \chi^{2} \geq 0.70\right)$ para conocer las correlaciones significativas: 1 ) entre las características de la muestra y sub-escalas de los cuatro tests, y 2) entre tests y entre sus sub escalas de los tests.

Las características de la muestra fueron: edad promedio 39.6 años; antigüedad promedio de 18.5 años; sexo: género femenino; categoría: enfermería; turno: matutino, y estado civil: casado.

\section{INSTRUMENTOS}

1) Test Clima Organizacional, construido por Caraveo J. et al., 1988, que mide: Cooperación, comunicación, recompensas, identificación con la empresa, estabilidad en el empleo, oportunidades de ascenso y relación con la autoridad.

Las características del cuestionario son las siguientes: es autoaplicable, cerrado, de 30 preguntas (tres opciones de respuesta: 2 puntos=bueno, 1 punto=regular y 0 punto=malo); escala rango para tres niveles de percepción de clima organizacional (adecuado $=60-50$, regular $=49-30$ e inadecuado $=29-0)$, con consistencia interna $(\alpha=.0 .87)$.

2) Test Motivación y Satisfacción Laboral. Elaborado, evaluado y estandarizado con personal hospitalario y supervisado por la Dirección General de Servicios de Salud del Gobierno del Distrito Federal (García, Ramírez y Pocas, 2006). Este cuestionario mide :a) Motivación a través de los indicadores de salario, prestaciones, seguridad, ambiente laboral y aspiraciones personales; $b$ ) satisfacción laboral a través de los indicadores de importancia del trabajo, expectativas de exigencias de éste, y expectativas entre esfuerzo y desempeño; $c$ ) sentido de posición y jerarquía a través de los indicadores de importancia del estatus o la jerarquía y reconocimiento de los demás hacia el cargo.

Las características del cuestionario son las siguientes: es autoaplicable, cerrado, de 35 preguntas (tres op- ciones de respuesta; 5 puntos=positivo, 3 puntos $=$ in termedio y 1 punto=negativo), escala rango para tres niveles de percepción de la motivación y satisfacción laboral (alto $=100-80$, mediano $=79-45$ y bajo $=44-0$ ). Presenta una confiabilidad en $S^{2} 1 / S^{2} 2>1.61$.

3) Maslach Burnout Inventory (MBI., Inventario construido por Maslach en 1981 y traducido y estandarizado en población mexicana por Grajales en 2001. Mide desgaste profesional a través de cuantificar disminución o pérdida de recursos emocionales de trabajadores quienes laboran con personas.

Las características del cuestionario son: autoaplicable, cerrado, de 22 preguntas (siete opciones de respuesta; 7 puntos=todos los días a 0 puntos=nunca), escala rango para tres niveles de percepción (alto, medio y bajo) en: Cansancio Emocional (CE), Despersonalización (DP) y Realización Personal (RP), con consistencia interna en sus tres subescalas y fiabilidad cercana a 0.90 .

4) Alcohol Use Disorders Identification Test (AUDIT). Financiado y supervisado por la OMS y realizado por Saunders et al., en 1993. Estandarizado por Saunders en 1993 para seis países, entre ellos México. Mide el Trastorno por Consumo de Alcohol (dependencia al alcohol, 303 y abuso del alcohol, 305), clasificado en DSM-IV R.Las características son: autoaplicable, cerrado, con 10 preguntas (cinco opciones de respuesta; 4 puntos=diario a 0 puntos=nunca), escala rango para tres niveles de percepción (consumo de bajo riesgo $=0-3$ puntos, consumo en riesgo $=4-7$ puntos y consumo en riesgo peligroso $>7$ puntos).

Nuestro procedimiento de investigación fue: se lanzó una convocatoria de encuesta, por medio de la repartición de volantes para invitar a todo trabajador de "base" a participar en encuesta laboral y psicometría, donde se informó acerca de los aspectos éticos de la investigación, puntualizando la aplicación voluntaria, anónima y confidencial.

La aplicación de encuesta y psicometría fue realizada dentro de áreas y jornadas laborales, individual o grupal (de acuerdo a las actividades laborales de cada trabajador). Fue ejecutada por estudiantes de una universidad privada de la carrera de Psicología, de octavo y noveno semestre, quienes fueron previamente instruidos en la aplicación de la misma, que contenía variables socio-demográficas-laborales y tests. 
El tiempo aproximado por aplicación de cada encuesta fue de 45 minutos; individual o grupal, de acuerdo a cada caso.

El tiempo de investigación fue de enero a mayo de 2010, con 80 intervenciones repartidas en los 20 hospitales, para abarcar los cuatro turnos.

El análisis de resultados fue realizado por la unidad de investigación de una universidad estatal.

En cuanto el análisis estadístico, se aplicó estadística descriptiva para las cuatro variables sociodemográficas, cuatro variables laborales para obtener las características de la muestra y estadística inferencial para medir el grado de correlación entre las características de ésta y los resultados de los cuatro tests, como la correlación entre los cuatro test y entre sus sub escalas. Se utilizó el manual y paquete estadístico SPSS para Windows, versión 17.

\section{RESULTADOS}

1) Estadística descriptiva: se obtuvieron las características de la muestra (ver Tabla 1) y porcentaje de las evaluaciones, las cuales son: trabajadores con clima organizacional inadecuado $N=1980 / 5,662=35 \%$; motivación e insatisfacción laboral bajo $N=1,874 / 5,662=$ $32.9 \%$; con síndrome de Burnout $N=1,548 / 5,662=$ $27.3 \%$, y con TCA $N=408 / 5,662=7.2 \%$.

2) Se utilizó estadística inferencial para obtener las correlaciones significativas de Chi-cuadrada de Pearson, p£ 0.05; $\chi 2^{3} 0.70$ existentes entre las características de la muestra y subescalas de los cuatro tests, reportándose sólo correlaciones significativas en la Tabla 2.

Tabla 1. Características de la muestra

\begin{tabular}{|c|c|c|c|c|c|c|c|}
\hline $\begin{array}{l}\text { Variables } \\
\text { sociodemográficas }\end{array}$ & \multicolumn{3}{|c|}{ Trabajadores "base" } & Variable laborales & \multicolumn{3}{|c|}{ Trabajadores "base" } \\
\hline \multicolumn{8}{|c|}{$\mathrm{n}=5,662$} \\
\hline Rango de edad & $N$ & $\%$ & media & Antigüedad laboral & $N$ & $\%$ & media \\
\hline 18.0 - 20 años & 140 & 2.5 & \multirow{5}{*}{$\begin{array}{l}39.6 \\
\text { años }\end{array}$} & $0.1-5$ años & 1638 & 28.9 & \multirow{6}{*}{$\begin{array}{l}18.5 \\
\text { años }\end{array}$} \\
\hline 20.1 - 30 años & 844 & 14.9 & & 5.1 - 10 años & 978 & 17.3 & \\
\hline 30.1 - 40 años & 1896 & 33.5 & & 10.1 - 15 años & 968 & 17.1 & \\
\hline 40.1 - 50 años & 1842 & 32.5 & & $15.1-20$ años & 792 & 14.0 & \\
\hline 50.1 - 60 años & 938 & 16.6 & & $20.1-25$ años & 1020 & 18.0 & \\
\hline & & & & $25.1-30$ años & 264 & 4.7 & \\
\hline Género & $N$ & \multicolumn{2}{|c|}{$\%$} & Localidad laboral & $N$ & \multicolumn{2}{|c|}{$\%$} \\
\hline masculino & 2712 & \multicolumn{2}{|c|}{47.9} & Distrito Federal & 3184 & \multicolumn{2}{|c|}{56.2} \\
\hline femenino & 2950 & \multicolumn{2}{|c|}{52.1} & Estado de México & 2478 & \multicolumn{2}{|c|}{43.8} \\
\hline Nivel académico & $N$ & \multicolumn{2}{|c|}{$\%$} & Categoría & $N$ & \multicolumn{2}{|c|}{$\%$} \\
\hline primaria & 940 & \multicolumn{2}{|c|}{16.6} & médico & 1164 & \multicolumn{2}{|c|}{20.6} \\
\hline secundaria & 1004 & \multicolumn{2}{|c|}{17.9} & área administrativa & 874 & \multicolumn{2}{|c|}{15.4} \\
\hline técnica & 1346 & \multicolumn{2}{|c|}{23.8} & enfermera & 1630 & \multicolumn{2}{|c|}{28.8} \\
\hline bachillerato & 754 & \multicolumn{2}{|c|}{13.3} & servicios básicos & 748 & \multicolumn{2}{|c|}{13.2} \\
\hline profesional & 1186 & \multicolumn{2}{|c|}{20.9} & asistente médica & 384 & \multicolumn{2}{|c|}{6.8} \\
\hline postgrado & 422 & \multicolumn{2}{|c|}{7.5} & Otros & 862 & \multicolumn{2}{|c|}{15.2} \\
\hline Estado Civil & $N$ & \multicolumn{2}{|c|}{$\%$} & Turno laboral & $N$ & \multicolumn{2}{|c|}{$\%$} \\
\hline casado & 3044 & \multicolumn{2}{|c|}{53.8} & Matutino & 3470 & \multicolumn{2}{|c|}{61.3} \\
\hline unión libre & 548 & \multicolumn{2}{|c|}{9.7} & Vespertino & 966 & \multicolumn{2}{|c|}{17.1} \\
\hline separado & 454 & \multicolumn{2}{|c|}{8.0} & Nocturno & 1112 & & \\
\hline divorciado & 388 & & & Jornada acumulada & 114 & & \\
\hline viudo & 192 & & & & & & \\
\hline soltero & 1036 & & & & & & \\
\hline
\end{tabular}

$N=$ número de trabajadores estudiados 
Tabla 2. Chi cuadrada de Pearson, correlaciones entre características de la muestra y subescalas de tests.

\begin{tabular}{|c|c|c|}
\hline Test & Variables: Características de la muestra y subescalas de los tests & $x^{2}$ \\
\hline Clima organizacional & $\begin{array}{l}\text { Antigüedad laboral (10.1 a } 15 \text { años) e identificación con la } \\
\text { empresa (inadecuada) }\end{array}$ & $0.710^{*}$ \\
\hline \multirow{3}{*}{$\begin{array}{l}\text { Motivación y } \\
\text { satisfacción laboral }\end{array}$} & Estado civil (casados) y Satisfacción laboral (bajo) & $0.849 *$ \\
\hline & Turno laboral (matutino) y Sentido de posición y jerarquía (bajo) & $0.870^{*}$ \\
\hline & $\begin{array}{l}\text { Antigüedad laboral (5.1 a } 10 \text { años) y Sentido de posición y } \\
\text { jerarquía (bajo) }\end{array}$ & $0.833 * *$ \\
\hline \multirow{2}{*}{$\mathrm{MBI}$} & Sexo (femenino) y con síndrome de Burnout & $0.880^{* *}$ \\
\hline & Categoría (medica) y con síndrome de Burnout & $0.970^{*}$ \\
\hline \multirow{2}{*}{ AUDIT } & Nivel académico (bachillerato) y con TCA & $0.851 *$ \\
\hline & Antigüedad laboral (5.1 a 10 años) y con TCA & $0.784^{* *}$ \\
\hline
\end{tabular}

* Correlación significativa a nivel 0.05 (2 colas)

${ }^{* *}$ Correlación significativa a nivel 0.01 ( 2 colas)

Nota: Alcohol Use Disorders Identification Test, AUDIT; Maslach Burnout Inventory, MBI.

3) También se utilizó estadística inferencial para obtener las correlaciones significativas de Chi-cuadrada de Pearson $\mathrm{p} \leq 0.05 ; \chi^{2} \geq 0.70$ existentes entre tests $\mathrm{y}$ entre sus subescalas de los tests, reportándose sólo correlaciones significativas en la Tabla 3:

Tabla 3. Prueba de Pearson, correlaciones significativas entre tests y entre sus subescalas de tests

\begin{tabular}{|c|c|c|c|}
\hline Variables tests & $\mathrm{X}^{2}$ & Variables subescalas de tests & $X^{2}$ \\
\hline \multirow{7}{*}{$\begin{array}{l}\text { Test de motivación y } \\
\text { satisfacción laboral y } \mathrm{MBI}\end{array}$} & \multirow{7}{*}{$-0.80 * *$} & Prestaciones (bajo) y Cansancio emocional (medio) & 0.772 \\
\hline & & Reconocimiento laboral.(poco) y Cansancio emocional (alto) & 0.843 \\
\hline & & Salario (bajo) y Despersonalización (medio) & 0.865 \\
\hline & & $\begin{array}{l}\text { Importancia del sentido de posición y jerarquía (bajo) y } \\
\text { Despersonalización (alto) }\end{array}$ & -0.712 \\
\hline & & $\begin{array}{l}\text { Reconocimiento de los demás hacia el cargo (bajo) y } \\
\text { Despersonalización (medio) }\end{array}$ & -0.929 \\
\hline & & Salario (bajo) y Falta de realización personal (alto) & 0.774 \\
\hline & & $\begin{array}{l}\text { Conflicto en ambiente laboral (alto) y Falta de realización } \\
\text { personal (alto) }\end{array}$ & 0.811 \\
\hline MBI y AUDIT & $0.74^{* *}$ & $\begin{array}{l}\text { Falta de realización personal (alto) y consumo de alcohol } \\
\text { (riesgo) }\end{array}$ & 0.745 \\
\hline
\end{tabular}

* Correlación significativa a nivel 0.05 ( 2 colas $)$

${ }^{* *}$ Correlación significativa a nivel 0.01 ( 2 colas)

Nota: Test Maslach Burnout Inventory, MBI, Alcohol Use Disorders Identification Test, AUDIT, 


\section{DISCUSIÓN}

Con base en el Plan de acción mundial sobre la salud de los trabajadores 2008-2017, (OMS, 2007) -en el que se insta a los Estados miembros en el apartado 1) a que elaboren planes de acción, en colaboración con los trabajadores, los empleadores y sus respectivas organizaciones, según proceda-, se realizó el presente estudio, que pretende puntualizar las condiciones laborales que se pueden considerar como factores de riesgo laboral porque afectan la salud mental de trabajadores de la salud en una institución representativa de salud pública, "la más grande de Latinoamérica", para tenerlo como base y así poder visualizar los puntos que requieren atención.

De acuerdo con los resultados obtenidos, se observó la falta de identificación con la empresa en trabajadores que tienen de 10 a 15 años de antigüedad, indicador muy importante porque hay trabajadores que se muestran apáticos ante la misión de la institución. Dicha misión se encuentra plasmada en carteles de oficinas principales o directivas. Entonces, se puede inferir que existe un trastorno de comunicación entre jefes y trabajadores de "base", porque, según el autor, la misión de las instituciones deben ser asimilada por los trabajadores en sus primeros días de trabajo, lo que se conoce como "amor a la camiseta" (Savio, 2008).

Encontramos una correlación significativa entre el test de motivación y satisfacción laboral y los trabajadores que tienen las características siguientes: ser casados, con cinco a 10 años de antigüedad laboral y del turno matutino. La motivación, por ende la satisfacción laboral, es de gran importancia porque en ella se encuentran factores de eficiencia y calidad del desempeño del trabajo (García, Ramírez y Pocas, 2006).

En este estudio se encontraron siete correlaciones entre las subescalas del test de motivación y satisfacción laboral y MBI, donde se ve la influencia directa de estos dos factores. Algunos autores han estudiado estos factores desde el factor humano y han afirmando que el trabajador ocupa un tercio del día en actividad laboral y es económicamente productivo más de la mitad de su existencia, por lo que requiere ser reconocido día con día para que su actividad productiva continúe y encuentre el equilibrio emocional a través de sus logros (Hernández, Dickson y Fernández, 2008).
En nuestro estudio se focalizó al género femenino de la categoría médica que presenta síndrome de Burnout. Apoyando la anterior correlación, existen investigaciones sobre profesionales de la salud que señalan que "médicos y enfermeras son quienes se encuentran entre las diez primeras actividades profesionales en sufrir desgaste profesional en el ámbito cognitivo (desgaste profesional) por la actividad que desempeñan" (Casanueva, Di Martino, 1994). Con respecto a los médicos del género femenino, investigadores reportan la sobrecarga de trabajo en este sector, y dicen: "Las mujeres llevan años soportando más carga de trabajo que el hombre, lo que en ocasiones desencadena en una peor salud mental" (Rubio, 2008).

Existe una correlación entre síndrome de Burnout y TCA; ante esta situación podemos mencionar el estudio exhaustivo que realizan Ortega y López en 2004, en el que se comenta que "los trabajadores de la salud que sufren este padecimiento se encuentran en malas condiciones físicas y mentales, lo que va ocasionar conflictos laborales, bajo rendimiento y alto número de incapacidades laborales".

Nuestro estudio se dirigió a trabajadores con nivel académico bachillerato y antigüedad de entre cinco y 10 años, quienes presentan correlación con TCA. Al respecto, podemos mencionar que estudios epidemiológicos del Instituto Nacional de Psiquiatría (INP) (CONADIC) señalan que "el TCA han aumentado en los últimos diez años y "el frecuente consumo de alcohol se presenta hasta en $51.7 \%$ en bachillerato, nivel más alto en jóvenes estudiantes". El impacto de este frecuente consumo es el desarrollo del TCA, según estudios realizados (Morales, 2010; Aranda, Pandomo, Torres, Salazar, Alderete, 2006).

El alto nivel de TCA y sus trastornos asociados tuvo como consecuencia la modificación de la cláusula 40 del Contrato Colectivo de Trabajo, que se compromete a establecer programas tendientes a prevenir y dar tratamiento a este padecimiento.

Es oportuno comentar el estudio de Hernández, Dickinson y Fernández (2008), realizado en trabajadores de salud de la misma entidad, donde se dice que las personas que presentan SB son quienes tienen bajo niveles de Locus de Control y apoyo institucional, por lo que es necesario identificar los principales disparadores organizacionales que provocan dicho padecimiento. 
El presente estudio corrobora la influencia entre la baja motivación y los conflictos laborales, y que ambos son factores de riesgo laboral que pueden desencadenar frecuentemente desgaste profesional y el consumo de alcohol en riesgo.

\section{REFERENCIAS}

Agencia Reforma (14 de Septiembre de 2009,). Dan a IMSS 14\% menos. El Siglo de Torreón (en línea). Recuperado el 31 Agosto de 2011 de: http://www.periodicodigital.com.mx/index.php?option=com_cont ent $\&$ task=view\&id=97892\&Itemid $=67$

Álvarez Escobar, M .C. (2009, marzo). The Burnout syndrome and the intensive care nursery staff. Revista Médica Electrónica: Síndrome de Burnout y personal de salud, 31, 3. Recuperado el 18 de marzo de 2011 y disponible en la URL: http://www.revmatanzas.sld. cu/revista\%20medica/año\%202009/vol3\%202009/ tema09.htm

Álvarez, G. (1992). El constructo "clima organizacional" concepto, teoría y resultados relevantes. Revista Interamericana de Psicología Ocupacional, 11(1, 2) 121-173.

Aranda, B. C., Pandomo, M. M., Torres, L. M., Salazar, E., Alderete, R. M. (2006). Síndrome de Burnout y manifestaciones clínicas en médicos familiares que laboran en una institución de salud para trabajadores del estado. Psicología y Salud, 16(1) 15-21.

Belloch-G, S.L., Calabuig J.R., Gómez-Salinas, R. y Renovell, F.V. (2000). Síndrome de agotamiento profesional en médicos residentes de especialidades médicas hospitalarias. Ann Med Interna, 17,118-122.

Bustinza, A. A., López-Herce, C.A., Carrillo, A., VigilEscribano, M.D., N. de Lucas G. y Panadero, C. E. (2000). Situación de Burnout de los pediatras intensivistas españoles. Revista de Atención Primaria, 52, 5, 418-423. Recuperado el 01 septiembre de 2011, de: http://www.opas.org.br/gentequefazsaude/bvsde/ bvsacd/cd49/pediatras.pdf

Caballero Martín, M. Bermejo, F. Nieto, G. y Caballero, M. (2001). Prevalencia y factores asociados al Burnout en un área de salud. Revista de Atención Primaria. $27,5,313-317$.
Se sugiere la realización de estudios de prevención y desarrollo de estrategias de intervención para su manejo.

Caraveo, J., Ramírez, G., Villatoro, J., Richerand, A. \& Escobar, A. (1988). Diagnóstico de necesidades en el ámbito laboral. La psicología social en México II (pp. 304-308). México: Asociación de Psicología Social.

Carr, J. Schmidt, A. y Deshon, R. (2003). Climate perceptions matter. J. Applied Psychology, 88(4) 605-619. Recuperado el 31 Agosto de 2011, de:http://www. io.psy.msu.edu/deshon/Papers/Carr\%20et\%20al\%20 (2003)\%20-\%20Climate.pdf

Carreño, S. Medina-Mora, E. Martínez, N. Juárez, F. y Vázquez, L. (2006). Características organizacionales, estrés y consumo de alcohol en una empresa textil mexicana. Salud Mental, 29, 63-70.

Cebria, A.J. (2003). Commentary: Chronic Distress y Worker Burnout: Hypotheses About Causes y Classification. Atención Primaria, 31, 572-574.

Clark, A. Oswald, A. y Warr, P. (1996). Is job satisfaction U-shaped in age? Journal of Occupational and Organizational Psychology, 69, 57-81.

Consejo Nacional contra las Adicciones CONADIC, (1986). Programas contra el Alcoholismo y el Abuso de Bebidas Alcohólicas, el Tabaquismo y la Farmacodependencia. (pp.36-40). México: COMADIC.

Contrato Colectivo de Trabajo del Instituto Mexicano del Seguro Social 2007-2009. (2007). Cláusula 40: Faltas justificadas con posterioridad. (pp. 29-30). México: Instituto Mexicano del Seguro Social.

Encuesta Nacional de Adicciones, ENA. (2002). Dirección General de Epidemiología; Instituto Mexicano de Psiquiatría. (pp. 34-40). México: SecretarÍa de Salud. Recuperado el 1 Septiembre de 2011 de: http://www. inegi.gob.mx/prod_serv/contenidos/espanol/bvinegi/productos/continuas/sociales/salud/2004/ena02. pdf 
Encuesta Nacional de Adicciones, ENA. (2008). Dirección General de Epidemiología. Instituto Mexicano de Psiquiatría. (pp.59-64). México: Secretaría de Salud.

Franco, B. X. (1999). Nivel de síndrome de agotamiento en médicos, enfermeras y paramédicos. Revi. Mex. Puer. Pediat, 99, 6, 252-260.

Freudenberger, H.J. (1974). Staff Burnout. J Soc Issues, 30, 159-165.

García, C., Ramírez, L. \& Pocas, K. (2006). Test de Motivación y Satisfacción Laboral. Recuperado el 01 Septiembre de 2011, de: http://www.monografias. com/trabajos36/satisfaccion-laboral/satisfaccionlaboral5.shtml

González, J. \& López, S. (2008). Tratamientos para el trastorno por consumo de alcohol en trabajadores del Sector Salud. Revista de Psicología Social Aplicada, 18(2), 127-149. Recuperado el 01 Septiembre de 2011, de: http://dialnet.unirioja.es/servlet/listaarticulos? tipo_busqueda=EJEMPLARyrevista_busqueda= 1209 yclave_busqueda $=245002$

González, R. (2000). La motivación en las organizaciones. Su reflejo en el sistema empresarial cubano. Recuperado el 01 de Septiembre de 2011, de: http:// www.monografias.com/trabajos36/satisfaccionlaboral/satisfaccion-laboral4.shtml

Grajales, G. T. (2001). Estudio de validez factorial del Masclach Burnout Inventory (versión española) en población de profesionales mexicanos. Memorias del CIE. 2: 63-82.

Hernández V., Dickson, M. \& Fernández O. (2008). El síndrome de desgaste profesional Burnout en médicos mexicanos. Rev. Fac. Med. UNAM, 51(1), Facultad de medicina. México: UNAM. Recuperado el 31 Agosto de 2011 de: http://www.medigraphic.com/pdfs/facmed/un-2008/un081c.pdf

Informe de la Evaluación Específica de Desempeño 20102011.Valoración de la Información contenida en el Sistema de Evaluación del Desempeño (SED). Atención curativa eficiente. México: Instituto Mexicano del Seguro Social. Recuperado el 31 de Agosto de 2011 de:

http://www.imss.gob.mx/NR/rdonlyres/3A10FE75B0E7-4A5A-A8B4-A18DFA308622/0/1011_E002_ ejecutivo.pdf

Lara, R. M., Benítez, M. M., Fernández, G.I \& Zárate, A. A. (1996). Aspectos epidemiológicos del adulto mayor en el Instituto Mexicano del Seguro. Revista de Salud Pública, 38(6)448-457.

Ley del Seguro Social (1995). En Diario Oficial de la Federación. Articulo 89, fracción III. Última reforma publicada DOF 09-07-2009.

Ley del Seguro Social (2004). En Diario Oficial de la Federación. Decreto por el que se reforman y adicionan los artículos 277 D y $286 \mathrm{~K}$ de la Ley del Seguro Social. Recuperado el 01 Septiembre de 2011 de: http://www.diputados.gob.mx/LeyesBiblio/ref/lss/ LSS_ref05_11ago04.pdf

Ley del Seguro Social (2005). En Diario Oficial de la Federación. Apartado Organismos descentralizados y/o desconcentrados, Instituto Mexicano del Seguro Social. Decreto por lo que se adiciona a la Ley del Seguro Social. Recuperado el 01 de Septiembre de 2011, de: http://www.diariooficial.segob.gob.mx/index.php?year=2005\&month $=04 \&$ day $=29$

López, L.E., Rodríguez, M.J., López, C.J., Peralta-P. \& Munguía-M.C. (2007). Desgaste profesional en médicos familiares y su asociación con factores sociodemográfico y laboral. Revista Médica del Instituto Mexicano del Seguro Social. 45, 13-19. Recuperado el 10 Abril de 2010 de: http://edumed.imss.gob.mx/ edumed/rev_med/pdf/gra_art/A90.pdf

Maslach, C. \& Schaufeli, WB. (1981). Historical and conceptual development of Burnout. In: Maslach C, Schaufeli W.B. and Marek T. Professional Burnout recent developments in theory and research. (pp.1-16). Washington: Taylor and Francis.

Medina-Mora M. \& Echeverría, L. (2005). World Health Organization Global Status Report on Alcohol. (pp. 234-249). Ginebra.

Morales, D. (2010). El Burnout, el compañero incómodo. Revista Anestesia en México, 16(1). Recuperada el 01 Septiembre 2011 de: www.fmca.org.mx/art/art. php?id $=878$

Organización Internacional del Trabajo. (1993). El trabajo en el mundo. Ginebra, Suiza: Organización Internacional del Trabajo. 5(1)159-198.

Organización Internacional del Trabajo. (1984). Factores Psicosociales en el Trabajo: Naturaleza, incidencia $y$ prevención, Ginebra, Suiza: Organización Internacional del Trabajo. Recuperado el 01 de Septiembre de $2011 \mathrm{de}$ http://factorespsicosociales.com/biblioteca/ publicaciones/FPS-OIT-OMS.pdf 
Organización Internacional del Trabajo. (2002). Seguridad social: un nuevo consenso; Ampliación de la cobertura personal de la protección social. (Capítulo III, pp.127158). Ginebra, Suiza: Organización Internacional del Trabajo.

Organización Mundial de la Salud. (2007). Salud de los trabajadores: proyecto de plan de acción mundial. 60ª Asamblea Mundial de la Salud A60/20, pág. 2 Recuperado el 01 de Septiembre de 2011, de: http:// apps.who.int/gb/ebwha/pdf_files/WHA60/A60_20sp.pdf

Organización para la Cooperación Económica y el Desarrollo (2007). Reporte anual 2007. Statistics of hospital attention.

Ortega, R. C. \& López, R. F. (2004). Burnout o síndrome de estar quemado en profesionales sanitarios: previsión y perspectivas. International Journal Clinical and Health Psychology. 4(1), 137-160. Recuperado el 01 Septiembre de 2011 de: http://www.aepc.es/ijchp/ articulos_pdf/ijchp-100.pdf

Pardo, A. J., López, H. F., Moriña, M. M., Pérez, C.M., Freire, P. P. \& Fernández, L.R. (2002). ¿Estamos quemados en atención primaria? Médicos de Familia, (27 - 32). Recuperado el 01 Septiembre de 2011 de: www.samfyc.es/Revista/PDF/v3n4/04.pdf

Parker, P.C., Baltes, B.B., Young, A.S. Huff, W.J., Altmann, A.R. LaCost, , A.H. y Roberts E.J. (2003). Relationships between psychological climate perception and work outcomes, a meta-analytic. J. Organiza. Behav, 24, 389-416.

Pera, G. \& Serra-Prat, M. (2002). Prevalecía del síndrome del quemado y estudio de los factores asociados en los trabajadores de un hospital comarcal. Gaceta Sanitaria, 16, 480-486.

Rojas, S. R. (1998). Guía para las investigaciones sociales. (pp.304-308). México: Plaza y Valdez,

Rubio, V. (18 de Marzo de 2008,). Las mujeres llevan años soportando más carga de trabajo que el hombre, lo que en ocasiones desencadena en una peor salud mental. Periódico el Mar. Recuperado el 01 Septiembre de 2011 de: http://www.psiquiatria.com/ noticias/atprimaria_y_sm/35602/.

Saunders, J. B., Aasland, O.G., Amundsen, A. \& Grant M. (1993). Alcohol consumption and related problems among primary health care patients: WHO Collaborative Project on Early Detection of Persons with Harmful Alcohol Consumption I. Addiction, 88, 349-362.

Saunders, J.B., Aasland, O. G., Babor, T. F., De la Fuente J. R., \& Grant, M. (1993). Development of the Alcohol Use Disorders Identification Test (AUDIT): WHO collaborative project on early detection of persons with harmful alcohol consumption. II. Addiction, 88, 791-804.

Savio, S.A. (2008). Magíster en Terapia de Pareja y Familia de Nova Southeastern University, USA, Hologramática - Facultad de Ciencias Sociales UNLZ - 5(8), 1, 121- 138.

Transcripción de la comparecencia del director general del IMSS (2007), Maestro Juan Molinar Horcasitas, ante la comisión de seguridad social de la cámara de diputados. Recuperado el 8 de Julio de 2010, de: http://www.imss.gob.mx/NR/rdonlyres/6D067338265F-4212-B0A8-F5761D2EF055/0/JMHCOMPARECENCIA.DOC 